\title{
Fundamentos para el análisis económico del tabaquismo: la utilidad marginal y los umbrales de asimilación
}

\author{
Daniel Fuentes \\ Universidad de Zaragoza
}

\begin{abstract}
RESUMEN
La normativa vigente sobre el consumo de tabaco establece que «el derecho de la población no fumadora a respirar aire no contaminado por el humo del tabaco prevalece sobre el de las personas fumadoras» (Ley 28/2005). Aunque se prohíbe fumar en ciertos espacios se permite habilitar zonas para fumar en algunos de ellos y en particular «en cualquier lugar en el que, sin existir prohibición de fumar, su titular así lo decida» (Art. 8). La normativa define y reasigna el derecho de propiedad ambiental en favor de los no-fumadores. Desde este punto de vista, es relevante analizar la conveniencia social de maximizar la utilidad de una categoría de individuos sabiendo que esto requiere la exclusión de la otra categoría. En nuestro análisis consideramos que la capacidad de los individuos para tolerar el tabaco en el ambiente no sólo depende de cómo varíe su utilidad marginal (o desutilidad) sino también de los umbrales de consumo mínimo y de consumo óptimo que están dispuestos a tolerar o que desean realizar. Se obtiene una solución de tipo bang bang que oscila entre las situaciones de contaminación cero (exclusión de los fumadores) y de máximo consumo de tabaco (exclusión de los no-fumadores). En este caso el principio de equimarginalidad, paradigma del caso estándar de contaminación, conduce a una solución de segundo rango. También se muestra por qué puede resultar económicamente racional que en presencia de fumadores compulsivos los titulares de espacios privados opten voluntariamente por prohibir el consumo de tabaco.

Palabras clave: derechos de propiedad; externalidades; contaminación; bienes comunes; tabaquismo

Códigos JEL: H23, I18, D62
\end{abstract}




\section{INTRODUCCIÓN}

La Ley 28/2005 de medidas sanitarias frente al tabaquismo y reguladora de la venta, el suministro, el consumo y la publicidad de los productos del tabaco (BOE 27/12/2005) pretende ser «garantía de que el derecho de la población no fumadora a respirar aire no contaminado por el humo del tabaco prevalece sobre el de las personas fumadoras». En ella se establecen ciertas limitaciones al consumo de tabaco, además de otras medidas relativas a su publicidad y promoción. La Ley parte de la distinción entre lugares donde se establece la prohibición total de fumar y lugares donde se prohíbe fumar pero se permite la habilitación de zonas para fumar, siempre que se cumplan determinados requisitos, tales como una señalización adecuada, la separación física del resto de las dependencias y la dotación de sistemas de ventilación independiente. En particular se prohíbe totalmente fumar en centros de trabajo públicos y privados, salvo en los espacios al aire libre; en centros docentes y formativos, independientemente de la edad del alumnado y del tipo de enseñanza; y en instalaciones deportivas y lugares donde se desarrollen espectáculos públicos, siempre que no sean al aire libre (Ley 28/2005, Art. 7). Además se prohíbe fumar, aunque se permite habilitar zonas para fumar, «en cualquier otro lugar en el que, sin existir prohibición de fumar, su titular así lo decida» (Ley 28/2005, Art. 8). En suma, la normativa define y reasigna los derechos de propiedad sobre la calidad ambiental de ciertos espacios asociados al consumo de tabaco. Este trabajo pretende contribuir, desde la perspectiva del análisis económico, al debate sobre la reasignación de tales derechos y a la racionalidad económica de la normativa.

Una muestra de que el debate no es irrelevante puede encontrarse en el Estudio 2627 del Centro de Investigaciones Sociológicas según el cual al 50,2\% de la población le molesta «mucho» $\mathrm{o}$ «bastante», en términos generales, que las personas que le rodean fumen habitualmente mientras que al 49,4\% le molesta «poco»o «nada» (CIS 2005, pregunta 12). En el mismo estudio se afirma que el 48,3\% de la población prefiere salir con gente que no fume y, al mismo tiempo, que el 53,5\% está de acuerdo con la afirmación «fumar es un derecho que tiene la gente y no hay por qué limitarlo» (CIS 2005, pregunta 13). Por otra parte, como señala la propia Ley 28/2005 en la exposición de motivos «el tabaquismo es la primera causa aislada de mortalidad y morbilidad evitable».

Si se recurre a la literatura económica sobre la determinación del nivel óptimo de contaminación ambiental se observará que ésta es enorme y que se basa mayormente en los principios de equimarginalidad y de compensación entre los beneficios y los costes asociados a la contaminación. La verificación de ambos principios permite a la teoría económica demostrar que existe un nivel de contaminación óptimo que interna- 
liza el coste social asociado a la contaminación (Baumol y Oates, 1988). Sin embargo, en palabras de Field y Field (2003, p.118), «el modelo (...) es muy general y al presentarlo se corre el riesgo de dar una idea excesivamente simplista de los problemas de contaminación que aquejan al mundo real. (...) Los agentes que contaminan pueden ser de todo tipo y tamaño, y actuar en contextos económicos de toda clase. Hasta el estudio de las propiedades más sencillas de los costes de reducción de la contaminación en casos concretos exige enormes recursos.».

En el caso concreto de las externalidades asociadas al consumo de tabaco la teoría económica ha partido del supuesto de la soberanía del consumidor. Como recuerda López-Nicolás (2004) «algunos autores racionalizaron la decisión de fumar como una decisión de riesgo calculado» obviando que en el hábito de fumar existe un componente adictivo fundamental. Es por ello que la práctica económica ha propuesto modelos en los que, como en el presente trabajo, la adicción es compatible con la soberanía del consumidor (Becker y Murphy, 1988; Suranovic, Goldfarb y Leonard, 1999).

En lo que concierne al consumo de tabaco en espacios cerrados el modelo general de la contaminación (Pearce y Turner, 1995; Field y Field, 2003) no recoge explícitamente el caso en que el nivel mínimo de contaminación necesario para satisfacer la utilidad de los agentes fumadores que acceden a un local es superior al nivel de contaminación que maximiza la utilidad de los agentes no-fumadores. La diferencia principal entre el escenario general y el que consideramos aquí radica en que las restricciones sobre la capacidad de asimilación de la contaminación son mucho más exigentes en éste que en aquel, tanto que la mera presencia en el local de alguna de las dos categorías de individuos (fumadores, no-fumadores) impide la maximización de la utilidad de la otra. Más aún, la maximización de la utilidad de cualquiera de las dos categorías impide la cohabitación espacial de ambas ${ }^{1}$. Dicho de otro modo: maximizar la utilidad de los clientes no-fumadores requiere prohibir el consumo de tabaco en el local. La cuestión que afrontamos es si resulta óptimo maximizar la utilidad de una categoría de individuos sabiendo que esto requiere la exclusión de la otra categoría. Para responder a ésta y otras cuestiones partimos de la base que la capacidad de los individuos para tolerar el tabaco en el ambiente no sólo depende de cómo varíe su utilidad marginal (o desutilidad) sino también de los umbrales de consumo mínimo y de consumo óptimo.

El modelo presenta una solución de tipo bang bang (Chiang, 1984) que oscila entre las situaciones extremas de contaminación cero (exclusión de los individuos fumadores)

${ }^{1}$ Se sobreentiende que ambas categorías pueden cohabitar pero a costa de que ambas renuncien forzosamente a maximizar su utilidad. 
y máximo consumo de tabaco (exclusión de los individuos no-fumadores). Variaciones en la asimilación de la reducción del consumo de tabaco (la capacidad de abstenerse de fumar pero también las mejoras en los sistemas de ventilación de espacios cerrados) pueden hacer oscilar el óptimo social de uno a otro extremo, haciendo que la propiedad de la calidad ambiental de un local recaiga en una u otra categoría de individuos y que el local sea declarado «exclusivamente fumador» o «exclusivamente no-fumador». El resultado más relevante consiste en mostrar cómo, en presencia de fumadores muy exigentes o dependientes, el equilibrio óptimo puede pasar de la situación de máximo consumo de tabaco a la de calidad ambiental óptima. La explicación a este resultado reside en la modelización de la adicción al tabaco, que no interpretamos únicamente como un incremento en la utilidad marginal del consumo de tabaco sino también como una reducción en la capacidad de los fumadores para asimilar la calidad ambiental.

El artículo se estructura del siguiente modo. En la sección 1 se presenta la contaminación como problema económico y se exponen las particularidades del caso que nos ocupa. La sección 2 consiste en una aproximación gráfica e intuitiva al óptimo de contaminación a partir de lo expuesto en la sección precedente. La sección 3 trata sobre el modo de alcanzar dicho óptimo. En la sección 4 se presenta el enfoque analítico del modelo así como un ejemplo algebraico-numérico que ilustra la convivencia entre individuos fumadores y no-fumadores dentro de un mismo local en presencia de restricciones en la capacidad de asimilación del consumo de tabaco. Las principales conclusiones se presentan en la sección 5.

\section{LA CONTAMINACIÓN COMO PROBLEMA ECONÓMICO}

Para la teoría económica «la contaminación es un coste externo y sólo ocurre cuando uno o más individuos sufren una pérdida de bienestar (Pearce y Turner 1995, p.100).» Muchos manuales de economía ambiental tratan la determinación del óptimo de contaminación recurriendo al caso de una fábrica que contamina un río en cuyo curso bajo existe una piscifactoría. En el ejemplo la primera impone a la segunda un coste externo que guarda proporción con el nivel de contaminación generado. La piscifactoría (el agente que soporta el coste marginal externo) maximiza sus beneficios (o, alternativamente, minimiza el coste social externo) cuando la fábrica paraliza por completo su actividad. En los modelos más habituales se considera que el medio ambiente dispone de cierta capacidad natural para asimilar parte de la contaminación generada. En este caso la cohabitación de ambas actividades es factible aún cuando la piscifactoría se encuentre en el punto de optimización de sus beneficios. Por su parte cuando la fábrica (el 
agente que causa la externalidad) maximiza sus beneficios el daño causado a la piscifactoría es máximo, de modo que ésta puede verse forzada a paralizar su actividad. En estas condiciones la teoría económica afirma que existe un punto de equilibrio en el que se maximiza el interés colectivo y que permite la cohabitación de ambas actividades.

El principio de la equimarginalidad permite demostrar que existe un nivel de contaminación óptimo, desde el punto de vista colectivo, que internaliza el coste social externo (Baumol y Oates, 1988). Field y Field (2003, p.114) definen la equimarginalidad del siguiente modo: «si tenemos varias fuentes generadoras de una sustancia contaminante concreta con diferentes costes marginales de reducción y queremos reducir el nivel total de emisiones con el menor coste posible tendremos que repartir la reducción entre las diversas fuentes de modo que el coste marginal de reducción de todas ellas sea el mismo.» Además del principio de la equimarginalidad la determinación del óptimo de contaminación incluye el supuesto de compensación potencial entre los agentes económicos afectados (o criterio de Kaldor-Hicks) según el cual se puede hacer balance, al menos en teoría, entre los beneficios y los costes asociados a un nivel determinado de contaminación. En palabras de Azqueta (2005, pág. 184) «si la suma neta es positiva, los beneficiados podrían eventualmente compensar a los perjudicados, y la alternativa es, en principio, aceptable.»

En ausencia poco probable de costes de transacción el problema causado por la contaminación podría resolverse por medio de la negociación directa entre contaminados y contaminadores. En un artículo de referencia Coase (1960) mostraba cómo la negociación directa permitiría alcanzar el óptimo de contaminación independientemente de a favor de quién se definiesen los derechos de propiedad sobre el medio: el «contaminado» o el «contaminador». La solución de referencia es la conocida como «paradigma pigouviano» (Baumol y Oates, 1988), por el cual se define la fiscalidad que internaliza el coste externo asociado a la contaminación. Esta tasa «es igual al coste marginal externo (esto es, el daño causado por una unidad marginal de contaminación) en el nivel óptimo de contaminación (Pearce y Turner 1995, p.120)».

Sin embargo en la realidad se observan situaciones en las que el nivel óptimo de contaminación no se alcanza, o bien por exceso (situaciones de sobreexplotación ambiental) o bien por defecto (subexplotación o deficiente puesta en valor de recursos ambientales). Ambas situaciones son asociadas en la literatura económica al problema de los bienes comunes (o de sobreexplotación) y al de los anticomunes (o de subexplotación) respectivamente, si bien la evidencia empírica de éste último problema no parece alarmante a ojos de la literatura y por ello su análisis teórico no ha sido objeto de contribuciones mayores (Buchanan y Yoon, 2000). 
Consideremos dos individuos que comparten un mismo espacio, por ejemplo un local cerrado. Sólo uno de ellos es fumador y su función de utilidad depende del consumo de cigarrillos (q). Como es habitual en la literatura suponemos que ésta es cóncava y tiene un máximo donde el consumo de tabaco alcanza un valor que denotamos $\mathrm{q}^{\mathrm{F}}$. El individuo no-fumador sufre una desutilidad o coste creciente con respecto al consumo de tabaco $^{2}$. De acuerdo con la literatura este coste es convexo (Riera et al. 2005, pág. 11). En la Gráfica 1 se representan tanto la utilidad marginal del fumador (UMa F) como el coste marginal externo que soporta el no-fumador (CMa NF) en función del consumo de tabaco. Se aprecia cómo el no-fumador es capaz de asimilar un nivel de contaminación máximo denotado $\mathrm{q}^{\mathrm{NF}}$, aunque con merma en su utilidad. Más allá de este umbral la desutilidad del no-fumador es tan elevada que opta por abandonar el local. A partir de este extremo, el coste total que soporta el no-fumador es menor a medida que el consumo de tabaco disminuye $\left(\mathrm{q} \leq \mathrm{q}^{\mathrm{NF}}{ }_{0}\right)$. Cuando el nivel de contaminación se reduce a $\mathrm{q}^{\mathrm{NF}}$ el no-fumador minimiza su desutilidad. A partir de este punto cualquier mejora de la calidad ambiental deja de tener efectos positivos en el bienestar del individuo no-fumador, por lo que $\mathrm{q}^{\mathrm{NF}}$ representa el máximo consumo de tabaco que no deteriora la utilidad del no-fumador. En la Gráfica 1 también se observa que cuanto mayor es el consumo de tabaco mayor es la utilidad del fumador, que alcanza un máximo en el punto $\mathrm{q}^{\mathrm{F}}$. A partir de este nivel cualquier incremento del consumo de tabaco carece de efectos positivos sobre la utilidad de ambos individuos.

En el modelo «general» (Pearce y Turner, 1995; Field y Field, 2003) se considera que el nivel de contaminación mínimo sin el cual es imposible el desarrollo de la actividad del contaminador es igual al nivel de contaminación que maximiza los beneficios del contaminado (o inferior, si se tiene en cuenta la capacidad del medio ambiente para asimilar cierto nivel de contaminación). Este nivel mínimo de contaminación se representa habitualmente en el origen de coordenadas, por lo que se tiene $\mathrm{q}^{\mathrm{NF}} \geq \mathrm{q}^{\mathrm{F}}=0$. El modelo general no recoge el caso en que el nivel mínimo de contaminación necesario

2 Podría considerarse que el individuo no-fumador maximiza su propia función de utilidad en lugar de minimizar una función de desutilidad o coste, como en el caso paradigmático de la fábrica y la piscifactoría. Sin embargo, eso supondría considerar una utilidad máxima positiva cuando el consumo de tabaco es nulo y nula cuando éste es cero. En realidad fumar supone una utilidad al fumador y una desutilidad al no-fumador, y no fumar supone una desutilidad al fumador y una ausencia de desutilidad (coste cero) al no-fumador. Esto es lo que la literatura entiende por un «mal» en lugar de un «bien» (Riera et al. 233). El análisis formal es sustancialmente el mismo en ambos casos. 
para desarrollar la actividad contaminadora es superior al nivel de contaminación con el que el contaminado maximiza sus beneficios. Es decir, que el fumador requiere un consumo mínimo de tabaco $\left(\mathrm{q}_{0}^{\mathrm{F}}\right)$ superior al máximo consumo que no deteriora la utilidad del no-fumador ( $\left.\mathrm{q}^{\mathrm{NF}}\right)$ : $0 \leq \mathrm{q}^{\mathrm{NF}} \leq \mathrm{q}^{\mathrm{F}}$. Esta situación se ilustra en la Gráfica 2: el fumador entra el local si se le permite fumar como mínimo $\mathrm{q}_{0}^{\mathrm{F}}$ cigarrillos, lo que necesariamente va a mermar la utilidad del no-fumador. Además el análisis habitual de la contaminación tampoco considera el caso en que el nivel de contaminación que maximiza los beneficios del contaminador es mayor que el máximo nivel de contaminación tolerable por el contaminado, de modo que el no-fumador se ve forzado a abandonar el local incluso antes de que el fumador haya alcanzado el máximo de su utilidad $\left(\mathrm{q}^{\mathrm{F}}>\mathrm{q}_{0}^{\mathrm{NF}}\right)$.

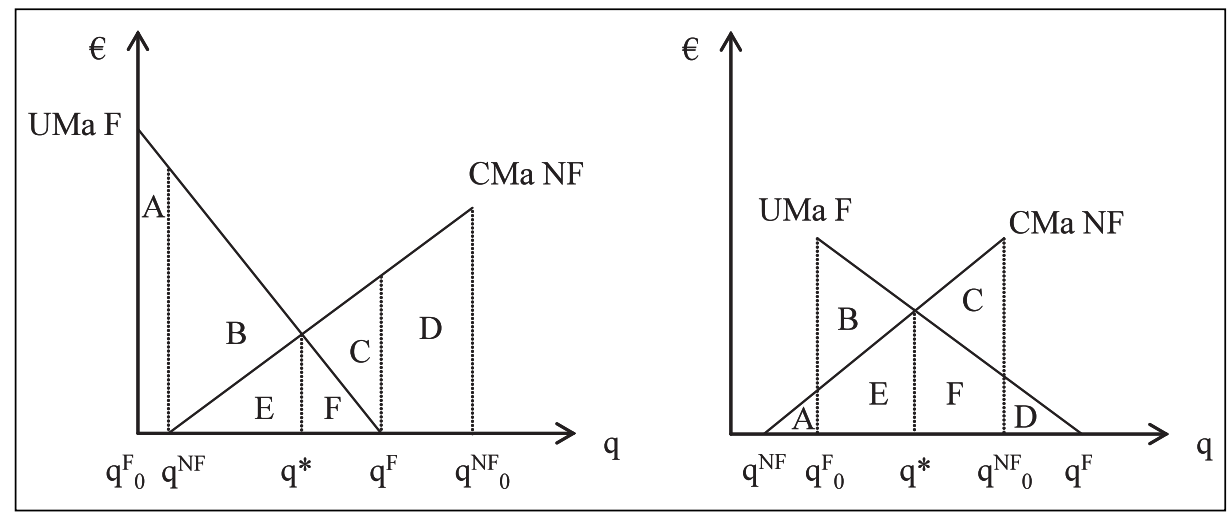

Gráfica 1

Gráfica 2

La diferencia entre el escenario general (Gráfica 1) y el que consideramos aquí (Gráfica 2) radica en los umbrales de asimilación del consumo de tabaco por parte de los individuos fumadores y no-fumadores. Field y Field (2003, págs. 98-99) señalan al respecto lo siguiente: «La presunción sobre la existencia o inexistencia de umbrales en las funciones de daño (...) es clave para entender las políticas de control de la contaminación que se aplican en la realidad. La cuestión de si las funciones de daño de algunas sustancias presentan un umbral ha sido objeto de intensos y continuos debates.» 


\section{EL ÓPTIMO DE CONTAMINACIÓN EN PRESENCIA DE RESTRICCIONES EN LA CAPACIDAD DE ASIMILACIÓN: UNA APROXIMACIÓN INTUITIVA}

De acuerdo con el principio de equimarginalidad anteriormente citado el óptimo de contaminación, que denotamos $\mathrm{q}^{*}$, es aquel en que se verifica la igualdad de las utilidades marginales de ambos individuos. En el caso general el óptimo aporta un nivel de bienestar más elevado que cualquier otro nivel de contaminación comprendido en el intervalo $\left(\mathrm{q}_{0}^{\mathrm{F}}, \mathrm{q}^{\mathrm{NF}}{ }_{0}\right)$. El razonamiento es bien conocido (Baumol y Oates, 1988; Pearce y Turner, 1995). Ahora bien, ¿qué ocurre cuando consideramos un consumo de tabaco tal que sólo uno de los dos agentes está dispuesto a acceder al local bien porque la concentración de tabaco en el ambiente es intolerable para el no-fumador $\left(\mathrm{q}>\mathrm{q}^{\mathrm{NF}}{ }_{0}\right)$, bien porque las exigencias sanitarias y ambientales son consideradas como excesivamente duras por el fumador $\left(\mathrm{q}<\mathrm{q}_{0}^{\mathrm{F}}\right)$ ?

Consideremos nuevamente la Gráfica 2. El bienestar asociado al consumo de tabaco en cantidad $\mathrm{q}^{*}$ se corresponde con el saldo entre la utilidad que reporta al fumador y la desutilidad que genera al no-fumador: $\mathrm{W}^{*}=(\mathrm{B}+\mathrm{E})-(\mathrm{A}+\mathrm{E})=\mathrm{B}-\mathrm{A}$. Por otra parte el bienestar que corresponde a la minimización de la desutilidad del no-fumador es nulo: $\mathrm{W}^{\mathrm{NF}}=0$, ni el fumador disfruta del tabaco ni el no-fumador soporta coste alguno. Entonces si $\mathrm{B}<\mathrm{A}$ el consumo óptimo de tabaco dejará de ser $\mathrm{q}^{*}$ para situarse en $\mathrm{q}^{\mathrm{NF}}$, de acuerdo con el principio de compensación potencial. En el caso opuesto el bienestar corresponde a la superficie $\mathrm{W}^{\mathrm{F}}=(\mathrm{B}+\mathrm{E}+\mathrm{F}+\mathrm{D})-(\mathrm{A}+\mathrm{E}+\mathrm{F}+\mathrm{C})=(\mathrm{B}-\mathrm{A})+(\mathrm{D}-\mathrm{C})$, es decir al saldo entre la máxima utilidad del fumador y el máximo coste del no-fumador. $\mathrm{Al}$ igual que el caso anterior, si $\mathrm{D}>\mathrm{C}$ el óptimo social se encuentra en $\mathrm{q}^{\mathrm{F}}$ en lugar de $\mathrm{q}^{*}$ ya que $\mathrm{W}^{\mathrm{F}}>\mathrm{W} *>\mathrm{W}^{\mathrm{NF}}$ : resulta óptimo desde un punto de vista colectivo permitir al fumador que maximice su utilidad.

En realidad estamos frente a un panorama compuesto por distintos escenarios donde el más habitual en la literatura no es sino un caso particular. La Tabla 1 presenta de modo intuitivo los posibles óptimos de contaminación en función de los umbrales de consumo y tolerancia al tabaco por parte de fumadores y no-fumadores (Gráfica 2).

TABLA 1.-_Variación del bienestar y consumo óptimo de tabaco

\begin{tabular}{|c|c|c|c|c|}
\hline Escenario 1 & Escenario 2 & Escenario 3 & \multicolumn{2}{|c|}{ Escenarios 4 y 5} \\
\hline \multirow{2}{*}{ B $>$ A y $D<C$} & \multirow{2}{*}{$\mathrm{B}>\mathrm{A}$ y $\mathrm{D}>\mathrm{C}$} & \multirow{2}{*}{$\mathrm{B}<\mathrm{A}$ y $\mathrm{D}<\mathrm{C}$} & \multicolumn{2}{|c|}{$\mathrm{B}<\mathrm{A}$ y $\mathrm{D}>\mathrm{C}$} \\
\hline & & & $\mathrm{A}+\mathrm{C}>\mathrm{B}+\mathrm{D}$ & $\mathrm{A}+\mathrm{C}<\mathrm{B}+\mathrm{D}$ \\
\hline $\mathrm{q}^{*}$ & $q^{F}$ & $q^{N F}$ & $q^{N F}$ & $\mathrm{q}^{\mathrm{F}}$ \\
\hline
\end{tabular}


Partamos de una situación en la que el nivel de contaminación socialmente óptimo verifica el principio de equimarginalidad (escenario 1): q*. ¿Qué ocurre si en esta situación consideramos un incremento en la pendiente de la utilidad marginal del fumador? La intuición nos indica que, ceteris paribus, las áreas B y D se incrementan y el área C se reduce hasta que, alcanzada una cierta pendiente, el punto $\mathrm{q}^{*}$ deja de maximizar el excedente social en beneficio del nivel de contaminación $\mathrm{q}^{\mathrm{F}}$ (escenario 2). La interpretación económica del incremento de la pendiente de la utilidad marginal del fumador es la siguiente: a mayor pendiente el coste marginal de reducir el consumo de tabaco se incrementa, es decir que privarse de un cigarrillo adicional no sólo resulta costoso (esto es así puesto que la pendiente de la utilidad marginal es negativa) sino cada vez más costoso. Utilidades marginales con pendientes elevadas se corresponden con fumadores para los que reducir el consumo de tabaco es muy costoso. En el extremo opuesto, una pendiente horizontal se corresponde con aquel fumador al que no le cuesta en absoluto reducir el consumo de cigarrillos. En efecto, si a partir del escenario representado en la Gráfica 2 consideramos una reducción en la pendiente de la utilidad marginal (el fumador es capaz de privarse de cigarrillos adicionales con menor esfuerzo) se intuye que no sólo el área $\mathrm{C}$ se incrementa sino que las áreas $\mathrm{B}$ y D se reducen hasta un punto en que el nivel de contaminación óptimo pasa a ser $\mathrm{q}^{\mathrm{NF}}$ en lugar de $\mathrm{q}^{*}$ (escenario 3 ).

Sin embargo la capacidad de los fumadores para reducir el consumo de tabaco no siempre se limita al esfuerzo en la reducción del consumo de cigarrillos sino que también puede afectar a los umbrales de consumo mínimo $\left(\mathrm{q}_{0}^{\mathrm{F}}\right)$ y deseado $\left(\mathrm{q}^{\mathrm{F}}\right)$. Al fumador le sucede lo que a un industrial contaminador, que no sólo le afecta el coste marginal de reducción de la contaminación sino el umbral mínimo de contaminación necesario para emprender su actividad $\left(\mathrm{q}_{0}^{\mathrm{F}}\right.$ ó contaminación sine qua non) y el umbral en que maximiza sus beneficios $\left(q^{\mathrm{F}}\right)$. Tomemos como punto de partida el escenario 2, en el que el incremento de la pendiente de la utilidad marginal del consumo de tabaco conduce al óptimo $\mathrm{q}^{\mathrm{F}}$. ¿Qué ocurre si al mismo tiempo se incrementa significativamente el consumo mínimo de cigarrillos exigido por el fumador? Intuitivamente se puede concluir que si el incremento de la superficie A es significativo la variación neta del excedente social es máxima cuando el consumo elegido es $\mathrm{q}^{\mathrm{NF}}$ (escenario 4). De igual modo podemos plantearnos qué ocurre cuando el umbral de consumo que maximiza la utilidad del fumador se incrementa. Si tomamos como referencia el escenario 3 y desplazamos considerablemente $\mathrm{q}^{\mathrm{F}}$ hacia la derecha (Gráfica 2) el aumento de la superficie D puede ser tal que resulte óptimo un consumo de tabaco igual a $\mathrm{q}^{\mathrm{F}}$ (escenario 5).

En la Gráfica 3 esquematizamos los cinco escenarios evocados. Su interpretación económica es la siguiente. En primer lugar, si el esfuerzo necesario para reducir el consumo de tabaco es muy elevado el óptimo social se incrementará hasta alcanzar el 
punto en que el fumador maximiza su utilidad $\mathrm{q}^{\mathrm{F}}$ (escenario 2). Esto también ocurre en el caso general de la contaminación: cuando se incrementa la pendiente de los beneficios marginales del contaminador el punto óptimo q* se desplaza hacia la derecha (Gráfica 1). En el caso general la explicación reside en que la capacidad de la economía para soportar la reducción de contaminación es débil (privarse de contaminación resulta demasiado costoso en comparación con la contribución que ésta aporta al proceso productivo), lo que en nuestro caso significa que el esfuerzo que ha de realizar el fumador para reducir el consumo de tabaco es tan elevado que resulta demasiado costoso en comparación con la desutilidad que éste genera a los no-fumadores. Si en estas circunstancias el fumador es capaz, con el mismo esfuerzo, de reducir drásticamente el umbral mínimo de cigarrillos entonces el consumo óptimo de tabaco pasaría de uno a otro extremo y se situaría en el punto en que el no-fumador minimiza su desutilidad $\mathrm{q}^{\mathrm{NF}}$ (escenario 4). En segundo lugar, si el esfuerzo necesario para reducir el consumo de tabaco es suficientemente bajo el óptimo social se alcanza privándose del consumo de tabaco y minimizando la desutilidad del no-fumador $\mathrm{q}^{\mathrm{NF}}$ (escenario 3). Sin embargo cuando el esfuerzo necesario para reducir el número de cigarrillos se combina con un incremento del umbral de consumo que maximiza la utilidad del fumador entonces es posible que el excedente social se maximice a costa del no-fumador (escenario 5).

GRÁfICA 3.-Capacidad para reducir el consumo de tabaco y óptimo social

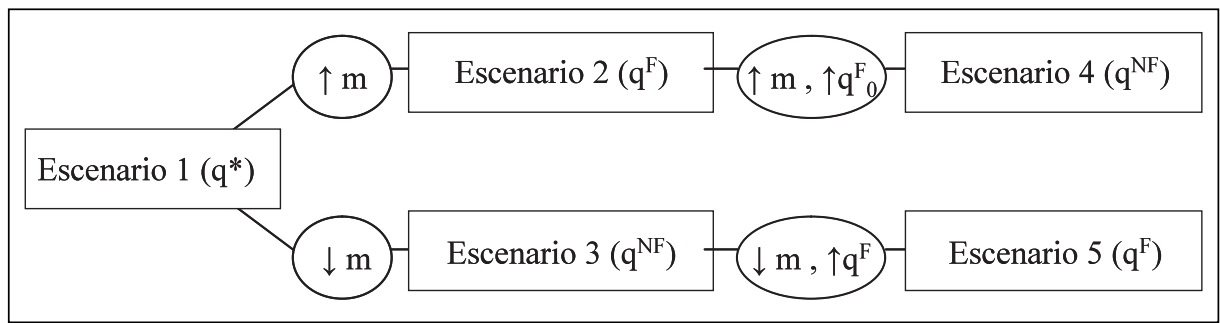

Nos hallamos pues ante un óptimo de contaminación que oscila entre uno y otro extremo $\left(\mathrm{q}^{\mathrm{NF}}, \mathrm{q}^{\mathrm{F}}\right)$. Únicamente en un caso particular (el escenario de referencia en los manuales de economía ambiental) encontramos un óptimo que permite la cohabitación entre fumadores y no-fumadores $\left(\mathrm{q}^{*}\right)$. 


\section{LA OBTENCIÓN DEL NIVEL ÓPTIMO DE CONTAMINACIÓN EN PRESENCIA DE RESTRICCIONES EN LA CAPACIDAD DE ASIMILACIÓN}

Existe una vasta literatura que analiza las distintas alternativas propuestas por la teoría económica para alcanzar el nivel óptimo de contaminación (Cropper y Oates, 1992). Además de la solución coasiana anteriormente citada la teoría económica muestra cómo se puede alcanzar el óptimo de contaminación utilizando impuestos, subvenciones, mercados de derechos y cuotas, todos ellos basados en el principio de equimarginalidad. A pesar de que los impuestos pigouvianos sobre la contaminación tienen propiedades de optimización «son la excepción, no la regla» (Pearce y Turner, 1995). Como señalan Field y Field (2003, pág. 214) «el objetivo real de este tipo de [impuestos] no es garantizar que los afectados sean indemnizados una vez ocurrido el daño (aunque esto sea importante), sino lograr que los posibles contaminadores sean más cuidadosos.» Algunas explicaciones al limitado papel de este tipo de impuestos pueden encontrarse en Pezzey (1988), entre las que destacan la incertidumbre sobre la equidad de los impuestos pigouvianos y la falta de conocimiento sobre la función del coste marginal externo de la contaminación.

En una situación como la descrita en el escenario 1 el óptimo de contaminación puede alcanzarse aplicando el paradigma pigouviano. Lo que nosotros tratamos de analizar es la consecución del nivel óptimo de contaminación económica en los restantes escenarios. Puesto que en nuestro caso el óptimo es del tipo bang bang las situaciones de no-optimalidad más relevantes son dos: i) el óptimo social se encuentra en el punto en que se maximiza la utilidad de los fumadores pero los derechos de propiedad sobre la calidad ambiental corresponden a los no-fumadores, ii) los derechos de propiedad ambiental corresponden a los fumadores pero el óptimo social exige que se minimice la desutilidad de los no-fumadores. La primera situación conduce a la subexplotación del la calidad ambiental: sería óptimo consumir $\mathrm{q}^{\mathrm{F}}$ cigarrillos y sin embargo el consumo se restringe a $\mathrm{q}^{\mathrm{NF}}$; mientras que en el segundo caso sucede lo contrario: el óptimo social consiste en reducir el consumo de cigarrillos a $\mathrm{q}^{\mathrm{NF}}$ y $\sin$ embargo se consumen en cantidad $\mathrm{q}^{\mathrm{F}}$.

La Gráfica 4 ilustra el segundo de los casos. El óptimo de contaminación se encuentra en el punto $\mathrm{q}^{\mathrm{NF}}$. Sin embargo cuando los derechos de propiedad ambiental pertenecen al individuo fumador éste pretenderá maximizar su utilidad aún a costa de generar una pérdida de bienestar equivalente al área $(\mathrm{A}-\mathrm{B})+(\mathrm{C}-\mathrm{D})>0$. Alternativamente, si consideramos el nivel de contaminación $\mathrm{q}^{*}$ el bienestar se incrementará con respecto a la situación de partida en una cuantía equivalente a la superficie $\mathrm{C}-\mathrm{D}>0$. Puesto que $\mathrm{A}>\mathrm{B}$ el punto $\mathrm{q}^{*}$ constituye un óptimo de segundo rango frente al óptimo de primer 
rango $\mathrm{q}^{\mathrm{NF}}$. En estas circunstancias puede afirmarse que una intervención pigouviana que tenga por objetivo alcanzar el nivel de contaminación $\mathrm{q}^{*}$ no será óptima. El razonamiento es el mismo si se considera una situación de subexplotación de la calidad ambiental.

La cuestión que se plantea es la de analizar las alternativas del sistema para corregir el exceso de contaminación, toda vez que la solución pigouviana que conduce al punto $\mathrm{q}^{*}$ resulta parcialmente satisfactoria ${ }^{3}$. Para alcanzar el óptimo de primer rango $\mathrm{q}^{\mathrm{NF}}$ será preciso o bien alterar la distribución inicial de los derechos de propiedad o bien imponer una norma de consumo máximo de tabaco al nivel $\mathrm{q}^{\mathrm{F}}$ (lo que en la realidad es equivalente a alterar la distribución inicial de la propiedad). En un mundo teórico sin costes de transacción podría considerarse la negociación directa, lo que no parece realista en el caso que nos ocupa. Obsérvese en la Gráfica 4 que una mínima reducción del consumo de tabaco a partir de $\mathrm{q}^{\mathrm{F}}$ provoca una merma de la utilidad del fumador sin reducir la desutilidad del fumador, que ya ha optado por abandonar el local. Se genera así un efecto disuasorio en el inicio de la hipotética negociación. En realidad la negociación coasiana equivale en este caso a que prevalezcan los derechos de los nofumadores. Mientras la propiedad ambiental corresponda a los fumadores el acuerdo entre ambas partes no parece posible, a menos que éstos renuncien voluntariamente al consumo de tabaco con la finalidad de evitar el coste social que se genera.

La teoría económica no suele recomendar la eliminación de la contaminación. El argumento habitual en la literatura es que, puesto que la contaminación se considera asociada a la actividad productiva, su nivel óptimo no ha de ser cero. Sin embargo la idea de contaminación cero no es absurda, como señalan entre otros Pearce y Turner (1995, pág. 100): «Hay al menos dos consideraciones que la hacen más razonable de lo que parece a primera vista. Estas son: a) el que el medio ambiente tiende a tener una capacidad de asimilación positiva, y b) el hecho de que es posible, hasta cierto punto, separar la actividad económica de los flujos de residuos que afectan al medio ambiente por medio de tecnologías reductoras de la contaminación.» La diferencia entre el caso general de contaminación asociada al sistema productivo y de las externalidades asociadas al consumo de tabaco reside en el hecho de que la producción difícilmente puede prescindir de la contaminación mientras que el fumador sí puede prescindir del tabaco,

3 Esto no significa en modo alguno que el regulador tenga que abandonar toda intervención pigouviana. Al contrario la solución de segundo rango sigue siendo satisfactoria ya que no sólo mejora el bienestar con respecto a la situación de partida y reduce el nivel de contaminación sino que permite la cohabitación de fumadores y no-fumadores. 
mejorando incluso su bienestar. La idea de contaminación cero no es excéntrica en el caso que nos ocupa.

GRÁFICA 4.—Propiedad ambiental de los fumadores y sobreexplotación

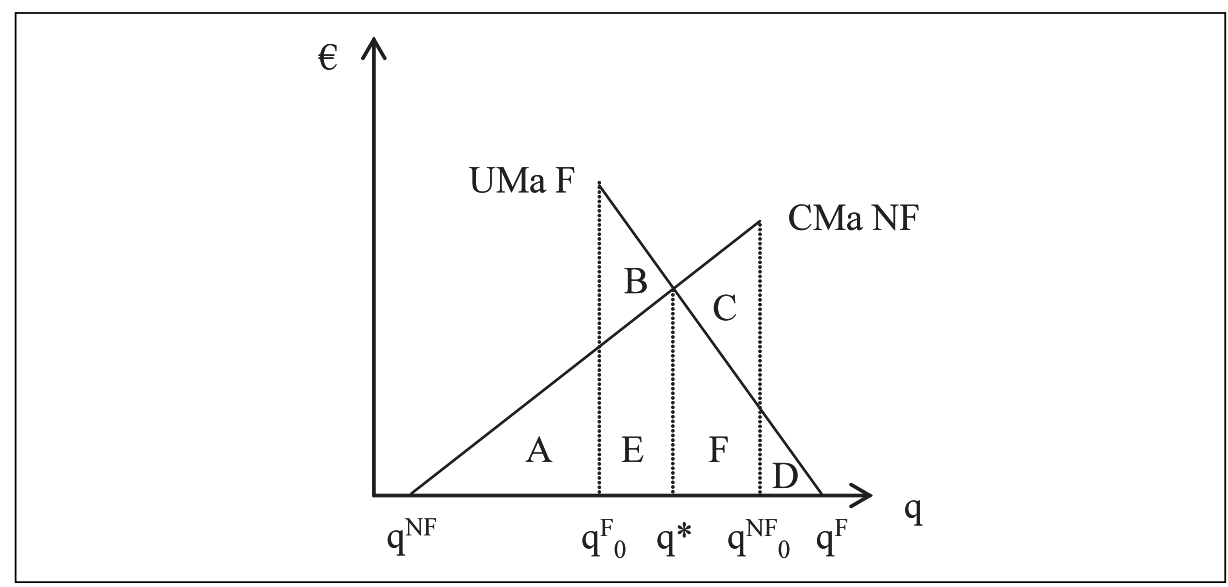

\section{El ENFOQUE ANALÍTICO: LA CONVIVENCIA ENTRE FUMADORES Y NO-FUMADORES}

Supongamos que la utilidad marginal que un fumador obtiene del consumo de $\mathrm{q}$ cigarrillos puede monetarizarse y se escribe $\operatorname{UMa} F(q)=m\left(q^{F}-q\right)$ donde $m>0$ indica la variación del esfuerzo marginal de reducción del consumo de cigarrillos ante una variación unitaria de q ( $m$ es la pendiente de la utilidad marginal del fumador $)^{4}$. Dicho de otro modo cuando el fumador decide privarse del consumo de un cigarrillo su utilidad marginal decrece en $m$ unidades monetarias. Por otra parte la desutilidad (o coste) marginal del no-fumador se escribe CMa NF $(q)=n\left(q-q^{N F}\right)$ donde $n>0$ es la pendiente de la desutilidad marginal del no-fumador (cuando el fumador incrementa

${ }^{4}$ Puesto que la utilidad del fumador es cóncava respecto al consumo de tabaco la utilidad marginal es creciente para cualquier $\mathrm{q}<\mathrm{q}^{\mathrm{F}}$. Podríamos escribir $\mathrm{UMa} \mathrm{F}=\mathrm{a}-\mathrm{mq}$ de manera general, donde a es la ordenada en el origen y $m$ la pendiente de la UMa F. Ahora bien, como sabemos que la utilidad es máxima en $\mathrm{q}^{\mathrm{F}}$ se verifica que la $\mathrm{UMa} F\left(\mathrm{q}^{\mathrm{F}}\right)=0$ por lo que $\mathrm{a}=\mathrm{m}^{\mathrm{F}}$. Es decir: $\mathrm{UMa} F=m\left(q^{\mathrm{F}}-\mathrm{q}\right)$. 
su consumo de cigarrillos en una unidad la utilidad marginal del no-fumador decrece en $n$ unidades monetarias $)^{5}$. Así al fumador que le cuesta mucho esfuerzo reducir su consumo de tabaco le corresponderá una $m$ muy grande, mientras que al no-fumador que tolere sin mucho esfuerzo cierta densidad de humo en su entorno le corresponderá una $n$ pequeña. De acuerdo con lo expuesto en las secciones precedentes suponemos que $\mathrm{q}^{\mathrm{NF}}<\mathrm{q}^{\mathrm{F}}{ }_{0}<\mathrm{q}^{*}<\mathrm{q}^{\mathrm{NF}}{ }_{0}<\mathrm{q}^{\mathrm{F}}$.

En el escenario comúnmente presentado en los manuales de economía ambiental, el óptimo se corresponde con el punto $\mathrm{q}^{*}$ tal que UMa F $\left(\mathrm{q}^{*}\right)=\mathrm{CMa} \mathrm{NF}\left(\mathrm{q}^{*}\right)$. Con esta formulación el consumo óptimo de tabaco toma la forma siguiente:

$$
q^{*}=\frac{m q^{F}+n q^{N F}}{m+n}
$$

En la Tabla 1 hemos presentado los distintos escenarios de contaminación en función de la capacidad de asimilación por parte de los individuos. Con la formulación aquí utilizada estamos en condiciones de caracterizar cada uno de dichos escenarios. Como hemos mencionado en las secciones precedentes, los escenarios 2 y 3 surgen cuando, a partir de la situación inicial en que $\mathrm{q}^{*}$ es óptimo, el esfuerzo marginal de reducir el consumo de cigarrillos se incrementa o se reduce, respectivamente. Para mayor claridad de la exposición denotamos $m_{1}$ la pendiente de la utilidad marginal del fumador tal que las superficies A y B en la Gráfica 2 son equivalentes. Del mismo modo denotamos $m_{2}$ la pendiente que verifica la igualdad de las áreas $\mathrm{C}$ y $\mathrm{D}, \mathrm{y} m_{3}$ la pendiente que verifica la condición $\mathrm{A}+\mathrm{C}=\mathrm{B}+\mathrm{D}$ :

$$
B>A \Leftrightarrow m>m_{1}=n \frac{\left(q_{0}^{F}-q^{N F}\right)\left(q^{*}-q^{N F}\right)}{\left(q^{F}-q_{0}^{F}\right)\left(q^{*}-q_{0}^{F}\right)}
$$

5 Puesto que el coste social o desutilidad es convexo el $C M a N F$ es creciente con respecto a q. De manera general podríamos escribir $C M a F=b+n q$, donde b es la ordenada en el origen y n la pendiente del $C M a F$. Ahora bien, como sabemos que el coste o desutilidad es nulo en $q^{N F}$ (éste es el umbral de tolerancia del no-fumador) se verifica que el $C M a N F\left(q^{N F}\right)=0$ por lo que $\mathrm{b}=-n q^{F}$. Es decir: $C M a N F=n\left(q-q^{N F}\right)$. 


$$
\begin{gathered}
C>D \Leftrightarrow m<m_{2}=n \frac{\left(q_{0}^{N F}-q^{N F}\right)\left(q_{0}^{N F}-q^{*}\right)}{\left(q^{F}-q_{0}^{N F}\right)\left(q^{F}-q^{*}\right)} \\
A+C>B+D \Leftrightarrow m<m_{3}=n \frac{\left(q_{0}^{F}-q^{N F}\right)\left(q^{*}-q^{N F}\right)+\left(q_{0}^{N F}-q^{*}\right)\left(q_{0}^{N F}-q^{N F}\right)}{\left(q^{F}-q_{0}^{N F}\right)\left(q^{F}-q^{*}\right)+\left(q^{*}-q_{0}^{F}\right)\left(q^{F}-q_{0}^{F}\right)}
\end{gathered}
$$

A la vista de las ecuaciones precedentes estamos en condiciones de afirmar que la negociación entre fumadores y no-fumadores se corresponde al escenario tipo 2 cuando la pendiente de la utilidad marginal del consumo de cigarrillos por parte del agente fumador verifica la desigualdad siguiente: $m>\max \left\{m_{1}, m_{2}\right\}$. En este caso el esfuerzo que ha de hacer el agente no-fumador para reducir su consumo de cigarrillos es tan elevado con respecto a la utilidad marginal que obtiene el no-fumador por la mejora de la calidad ambiental que resulta óptimo permitir que el primero maximice su utilidad aún a costa del segundo. Esto ocurre, por ejemplo, en los bares de copas donde individuos de ambas categorías disfrutan libre y conjuntamente de su ocio. También se puede considerar el caso inverso: cuando la pendiente de la utilidad marginal del consumo de cigarrillos por parte del agente fumador es tan pequeña en términos relativos que verifica la desigualdad $m<\min \left\{m_{1}, m_{2}\right\}$ entonces resulta óptimo restringir al máximo el consumo de tabaco. En este caso nos encontraríamos en una situación de tipo 3, lo que ocurre por ejemplo en los centros culturales, bibliotecas y museos.

TABLA 2.-Ó́ptimos de contaminación

\begin{tabular}{l|c|c|}
\multicolumn{1}{c}{} & \multicolumn{1}{c}{ Condición } & \multicolumn{1}{c}{ Optimo } \\
\cline { 2 - 3 } Escenario 1 & $\mathrm{m}_{2}>\mathrm{m}>\mathrm{m}_{1}$ & $\mathrm{q}^{*}$ \\
\cline { 2 - 3 } Escenario 2 & $\mathrm{m}>\max \left\{\mathrm{m}_{1}, \mathrm{~m}_{2}\right\}$ & $\mathrm{q}^{\mathrm{F}}$ \\
\cline { 2 - 3 } Escenario 3 & $\mathrm{m}<\min \left\{\mathrm{m}_{1}, \mathrm{~m}_{2}\right\}$ & $\mathrm{q}^{\mathrm{NF}}$ \\
\cline { 2 - 3 } Escenario 4 & $\min \left\{\mathrm{m}_{1}, \mathrm{~m}_{3}\right\}>\mathrm{m}>\mathrm{m}_{2}$ & $\mathrm{q}^{\mathrm{NF}}$ \\
\cline { 2 - 3 } Escenario 5 & $\mathrm{m}_{1}>\mathrm{m}>\max \left\{\mathrm{m}_{2}, \mathrm{~m}_{3}\right\}$ & $\mathrm{q}^{\mathrm{F}}$ \\
\cline { 2 - 3 } & &
\end{tabular}

La capacidad que tienen los individuos para asimilar el tabaco en el ambiente no sólo depende de cómo varíe su utilidad (o desutilidad) marginal sino también de los umbrales de consumo mínimo y de consumo óptimo: $\mathrm{q}_{0}^{\mathrm{F}}$ y $\mathrm{q}^{\mathrm{F}}$ respectivamente (Gráfica 3). Si esto no fuera así la diversidad de situaciones a las que nos enfrentamos quedaría reducida a los tres primeros escenarios mencionados en la Tabla 1. En realidad existen 
individuos fumadores más o menos dependientes del consumo de tabaco. Esto amplía el número de escenarios a considerar, si bien los posibles equilibrios son únicamente tres: $q^{*}, q^{F}$ ó $q^{N F}$. En la Tabla 2 exponemos las condiciones analíticas que describen cada uno de los cinco escenarios reseñados junto con los correspondientes óptimos de contaminación.

Así pues una variación relativa ${ }^{6}$ de la pendiente de la utilidad marginal del individuo fumador con respecto a la del no-fumador puede bastar para trasladar la situación desde el punto de equilibrio $\mathrm{q}^{*}$ a cualquiera de las soluciones extremas $\left(\mathrm{q}^{\mathrm{F}}\right.$ si $m$ crece lo suficiente, $\mathrm{q}^{\mathrm{NF}}$ si $m$ decrece). Las consecuencias no son neutras, pues una de las dos categorías de individuos puede verse forzada a abandonar el local. La inestabilidad del sistema es tal que a partir de una situación como la descrita en el escenario 2 es posible pasar a la descrita en el escenario 4 si el umbral mínimo de consumo de tabaco $\mathrm{q}_{0}{ }^{\mathrm{F}}$ crece lo suficiente como para que se verifique, ceteris paribus, la condición $\min \left\{m_{1}\right.$, $\left.m_{3}\right\}>m>m_{2}$. También en este caso el óptimo de contaminación requiere pasar de un extremo al otro. Lo mismo ocurre cuando a partir de una situación como la descrita en el escenario 3 se considera un incremento sustancial del umbral de máximo consumo de tabaco $\mathrm{q}^{\mathrm{F}}$ tal que se verifica la condición $m_{1}>m>\max \left\{m_{2}, m_{3}\right\}$ y se traslada el sistema a una situación como la descrita en el escenario 5.

GRÁFICA 5.-Convivencia entre fumadores y no-fumadores

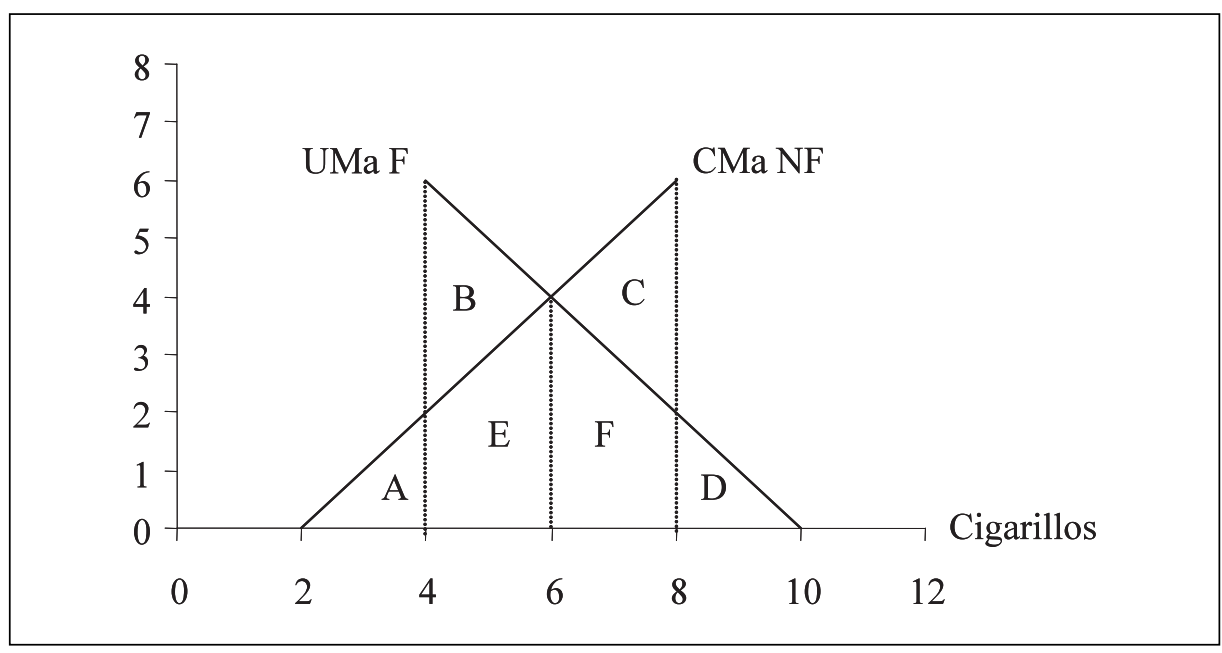

${ }^{6}$ Obsérvese que la variación de $m$ ha de ser relativa con respeto a $n$, de acuerdo con las expresiones $\mathrm{m}_{1}, \mathrm{~m}_{2} \mathrm{y} \mathrm{m}_{3}$ en las ecuaciones (2), (3) y (4). 
Para mayor claridad del análisis consideramos el ejemplo numérico representado en la Gráfica 5: el individuo no-fumador está dispuesto a tolerar sin ningún problema $q^{N F}$ $=2$ pitillos encendidos en el local. Sin embargo a medida que el consumo de tabaco se incrementa su utilidad marginal decrece con pendiente, en valor absoluto, $n=1$ hasta un consumo máximo de $q_{0}^{N F}=8$ cigarrillos. Alcanzado este consumo las molestias sufridas por el no-fumador en forma de picores en ojos y garganta le obligan a abandonar el local. Por otra parte el individuo fumador no entra al local si no se le permite fumar al menos $q_{0}^{F}=4$ cigarrillos. Su utilidad es óptima cuando puede consumir $q^{F}$ $=10$ cigarrillos en el tiempo que permanece en el local. La pregunta que pretendemos responder es ¿cuál es el consumo de cigarrillos que maximiza la utilidad conjunta de ambos categorías de individuos?

Resulta evidente que la formulación del problema depende del peso relativo de las utilidades marginales de los individuos, en el sentido de cómo de costoso sea el esfuerzo de reducción del consumo de tabaco $(m)$ con respecto a cómo de molesto sea el incremento de humo en el local $(n)$. Consideremos en un primer momento que ambos son equivalentes, es decir $n=m$. En esta situación es evidente que el consumo óptimo de tabaco es aquel que satisface a fumadores y no-fumadores por partes iguales. La ecuación (1) nos indica que la calidad ambiental que optimiza el bienestar social se corresponde con el punto $q^{*}=6$.

Consideremos ahora que se altera la relación de utilidades marginales entre ambas categorías de individuos, por ejemplo que el esfuerzo de reducir el consumo de tabaco es más costoso que el incremento de las molestias sufridas por los no-fumadores $(m>$ $n)$ ó viceversa $(m<n)$. Si la variación de $m$ es moderada el óptimo social seguirá permitiendo la cohabitación de ambas categorías, de acuerdo con lo enunciado en la Tabla 2 y conforme a la ecuación (1):

$$
q^{*}(m)=\frac{2+10 m}{1+m}
$$

En concreto las ecuaciones (2) y (3) nos permiten afirmar que cuando el valor de $m$ está comprendido entre 7/9 y 9/7 la convivencia entre ambas categorías de individuos no se ve comprometida y el consumo óptimo de tabaco en el local oscila entre 5,5 y 6,5 cigarrillos. Ahora bien si la variación de $m$ es sustancial entonces la convivencia entre fumadores y no-fumadores en el local puede verse comprometida. En nuestro ejemplo entendemos que $m$ varía de modo sustancial cuando alcanza valores superiores a 9/7 o se reduce a valores inferiores a 7/9. Así pues cuando $m>9 / 7$ el consumo óptimo de 
tabaco se corresponde con el consumo deseado por los agentes fumadores $\left(q^{\mathrm{F}}=10\right) \mathrm{y}$ los no-fumadores abandonan el local (escenario 2). Cuando $m<7 / 9$ el óptimo social requiere la optimización de la calidad ambiental, por lo que el consumo de tabaco se reduce al nivel $\mathrm{q}^{\mathrm{NF}} \leq 2$ y los fumadores abandonan el local (escenario 3). En ambos casos el nivel óptimo de contaminación está enfrentado con la convivencia de ambas categorías de individuos en el mismo local. Estamos frente a una solución del tipo bang bang cuando $m \notin(7 / 9,9 / 7)$.

Sin embargo el consumo óptimo de tabaco en el local no depende sólo de la pendiente de las utilidades marginales sino también de la relación entre los umbrales de consumo y tolerancia hacia el tabaco por parte de cada una de las dos categorías de individuos. En nuestro ejemplo el no-fumador es indiferente ante el consumo de uno o dos pitillos en el local y tolera el humo de hasta 8 cigarrillos (aunque estos supongan una merma creciente de su utilidad). Por otra parte el individuo fumador desea consumir entre 4 y 10 cigarrillos en el local. Consideremos por ejemplo el caso particular $m$ $=2$ y $n=1$. Esta relación entre las utilidades marginales se corresponde con la descrita en el escenario 2: se está afirmando que cuando al individuo fumador le cuesta mucho más prescindir de un pitillo adicional de lo que su consumo molesta no-fumador resulta óptimo desde el punto de vista social que se permita a los fumadores optimizar su utilidad (y en consecuencia maximizar el consumo de tabaco en el local). Ahora bien, ¿qué ocurre en esta situación si además el individuo fumador es muy dependiente del tabaco e incrementa el umbral mínimo $\mathrm{q}_{0}{ }^{\mathrm{F}}$ más allá de los cuatro cigarrillos inicialmente considerados? Dicho de otro modo, ¿qué sucede cuando el fumador abandona el local si no se le permite fumar al menos $\mathrm{q}_{0}{ }^{\mathrm{F}}+\Delta \mathrm{q}_{0}{ }^{\mathrm{F}}$ cigarrillos?

La respuesta a la anterior cuestión es contra intuitiva y por ello requiere una explicación. Podría pensarse que la exigencia o dependencia del individuo fumador refuerza la conclusión a la que se llega en el escenario 2: que el óptimo social se corresponde con la maximización de la utilidad de los individuos fumadores (bajo el argumento de que la satisfacción que reporta el consumo de tabaco es superior a la desutilidad sufrida por los no-fumadores). Si en la situación de partida $\left(\mathrm{q}_{0}{ }^{\mathrm{F}}\right)$ la utilidad social neta derivada del consumo de tabaco es positiva podría pensarse que también lo será, y con mayor razón, en presencia de fumadores compulsivos que, al menos en teoría, obtienen una mayor satisfacción del tabaco.

Pese a su aparente consistencia el razonamiento anterior es incorrecto en el siguiente punto: el individuo que incrementa el umbral mínimo $\mathrm{q}_{0}{ }^{\mathrm{F}}$ es tan dependiente del tabaco que no disfruta del local cuando la calidad ambiental pertenece al intervalo $\left(\mathrm{q}_{0}{ }^{\mathrm{F}}, \mathrm{q}_{0}{ }^{\mathrm{F}}+\Delta \mathrm{q}_{0}{ }^{\mathrm{F}}\right)$ cosa que sí hacen los individuos no-fumadores. Ocurre que si $\Delta \mathrm{q}_{0}{ }^{\mathrm{F}}$ es suficientemente grande la utilidad social neta del consumo de tabaco en el local pue- 
de ser negativa. Esto sucede cuando se verifica la condición $\mathrm{A}+\mathrm{C}=\mathrm{B}+\mathrm{D}$ (Gráfica 5) o analíticamente $m_{3}=m$ (ecuación 4). En nuestro ejemplo la utilidad social neta del consumo de tabaco es negativa cuando $\Delta \mathrm{q}_{0}{ }^{\mathrm{F}}>1,76$ : si el fumador exige o necesita consumir al menos $\mathrm{q}_{0}{ }^{\mathrm{F}}=5,76$ cigarrillos, y no más de 10 , entonces resulta óptimo restringir el consumo de tabaco y maximizar la calidad ambiental del local (ecuación 4). Podría afirmarse que la tabaco-dependencia acaba por perjudicar el disfrute del local por parte de los individuos fumadores, que verían cómo el local pasa a prohibir el consumo de tabaco.

No tiene que sorprender este resultado, aunque pueda parecer paradójico. El hecho de que el equilibrio óptimo pase de un extremo al otro (del máximo consumo de tabaco a la calidad ambiental óptima) en presencia de fumadores muy exigentes o dependientes ocurre porque en este modelo la tabaco-dependencia no se interpreta únicamente como un incremento en la utilidad marginal del consumo de tabaco $(m>n)$ sino también como una reducción en la capacidad de asimilación de la calidad ambiental por parte de los individuos fumadores: en realidad cuando se incrementa $\mathrm{q}_{0}{ }^{\mathrm{F}}$ los individuos fumadores están renunciando parcialmente al disfrute del local en beneficio de los individuos no-fumadores (es fácil intuir la satisfacción de los no-fumadores que acceden a un local al que renuncian a entrar los fumadores compulsivos).

Algo similar ocurre cuando el individuo fumador incrementa el umbral de consumo máximo de tabaco: $\mathrm{q}^{\mathrm{F}}>10$. Pongamos que estamos en el escenario 3 , donde una reducción de $m$ ha trasladado el óptimo social desde $\mathrm{q}^{*}$ hasta $\mathrm{q}^{\mathrm{NF}}$, por ejemplo $m=$ 0,5. Cuando el incremento de $\mathrm{q}^{\mathrm{F}}$ es sustancial (un fumador compulsivo) es posible que privarle del consumo de tabaco le provoque mayor desutilidad que la provocada al individuo no-fumador cuando al primero se le permite maximizar el consumo de tabaco. En nuestro ejemplo cuando $\mathrm{q}^{\mathrm{F}}>12,49$ cigarrillos (Tabla 2 - escenario 5- y ecuación 4), la satisfacción del fumador compensa las molestias del fumador y el equilibrio óptimo pasa de un extremo al otro (de la calidad ambiental óptima al máximo consumo de tabaco).

Finalmente consideramos los dos casos extremos paradigmáticos. En primer lugar el caso en que el individuo no-fumador es completamente insensible al consumo de tabaco en el local $(n \rightarrow 0)$. Resulta evidente que en este caso el consumo óptimo de tabaco es aquel que maximiza la utilidad de los fumadores ( $\mathrm{y}$ al mismo tiempo no deteriora la de los no-fumadores). Éste puede ser el caso de un bar de copas atestado de tabaco y en el que conviven fumadores y no-fumadores. De acuerdo con la ecuación (1) y los resultados generales en la Tabla 2 el óptimo en nuestro ejemplo se encuentra en $\mathrm{q}^{*}=10$ cigarrillos. Cuando se considera el caso extremo opuesto $(n \rightarrow \infty)$ la desutilidad sufrida por los individuos no-fumadores crece tan desmesuradamente con cada nuevo pitillo 
que la mejor alternativa consiste en restringir el consumo de tabaco a $q \leq 2$ cigarrillos: $\lim _{n \rightarrow \infty} q^{*}=2$. Esto ocurre por ejemplo en los ascensores y cabinas de teléfono, donde la restricción del consumo de tabaco maximiza el bienestar social.

\section{Conclusiones}

La LEY 28/2005 de medidas sanitarias frente al tabaquismo reconoce implícitamente a los individuos no-fumadores el derecho de propiedad sobre la calidad ambiental de ciertos espacios (centros de trabajo, centros docentes, instalaciones deportivas,...) y abre la posibilidad de reasignar la propiedad sobre la calidad ambiental en otros.

Analizamos los fundamentos económicos de estas medidas centrándonos en el papel de la capacidad de asimilación de la contaminación, que depende de: i) la relación entre las pendientes de las utilidades marginales de cada una de las dos categorías de individuos afectados (fumadores y no-fumadores); y ii) los umbrales de asimilación del consumo de tabaco (consumo mínimo exigido por los fumadores y contaminación máxima tolerada por los no-fumadores). La diferencia entre el modelo general de la contaminación y el caso aquí considerado reside en que las restricciones sobre la capacidad de asimilación de la contaminación son mucho más exigentes en éste: la maximización de la utilidad de cualquiera de las dos categorías impide la cohabitación espacial de ambas (la cohabitación es posible si y sólo si ambas renuncian a maximizar su utilidad).

- En lugar de la solución clásica que permite la cohabitación entre contaminadores y contaminados se obtiene una solución de tipo bang bang que oscila entre las situaciones de contaminación cero (exclusión de los individuos fumadores) y de máximo consumo de tabaco (exclusión de los individuos no-fumadores). Las primeras reducciones del nivel de contaminación son poco costosas para el fumador pero aún así no recibe compensación alguna.

- Variaciones en la asimilación de la reducción del consumo de tabaco (la capacidad de abstenerse de fumar pero también las mejoras en los sistemas de ventilación de espacios cerrados) pueden hacer oscilar el óptimo social de uno a otro extremo, haciendo que la propiedad de la calidad ambiental de un local recaiga en una u otra categoría de individuos y que el local sea declarado «exclusivamente fumador» o «exclusivamente no-fumador». Este resultado parece sustentar el hecho de que la normativa faculte a los bares, restaurantes y demás establecimientos de restauración cerrados de menos de $100 \mathrm{~m}^{2}$ a de- 
finir y reasignar la propiedad de la calidad ambiental, así como en general a «cualquier otro lugar en el que, sin existir prohibición de fumar, su titular así lo decida».

- Cuando al individuo fumador le cuesta mucho más prescindir de un cigarrillo adicional de lo que su consumo molesta al no-fumador resulta óptimo desde el punto de vista social que se permita a los fumadores optimizar su utilidad (y en consecuencia maximizar el consumo de tabaco en el local).

Ahora bien, ¿qué ocurre en esta situación si además el individuo fumador es muy dependiente del tabaco e incrementa significativamente el umbral de consumo mínimo exigido (amenazando con abandonar el local si no se le permite fumar más)? La respuesta es contra-intuitiva. Podría pensarse que la exigencia o dependencia del individuo fumador refuerza la conclusión anterior (bajo el argumento de que la satisfacción que reporta el consumo de tabaco a individuos muy dependientes es superior a la desutilidad sufrida por los individuos no-fumadores). Sin embargo, la exigencia del individuo que incrementa el umbral mínimo de consumo de tabaco está en realidad reduciendo sus posibilidades de disfrute del local en beneficio de individuos no-fumadores. Dicho de otro modo, puede resultar económicamente racional que se prohíba el consumo de tabaco en presencia de fumadores compulsivos.

Posibles extensiones al presente análisis pasan por considerar incentivos a la innovación, entendida ésta como mejora en la capacidad de asimilación de la contaminación: bien modificando la relación entre las pendientes de las utilidades marginales (reducción de la satisfacción asociada al consumo de tabaco, incremento de la utilidad asociada a la calidad ambiental), bien mejorando los umbrales de asimilación del consumo de tabaco (reducción de la adicción al tabaco, mejoras en los sistemas de ventilación).

\section{REFERENCIAS}

AzQueta, D. (2005), Introducción a la economía ambiental, Madrid: McGraw-Hill.

Baumol, W. y OAtes, W. (1988), The Theory of Environmental Policy, Cambridge, Cambridge University Press, $2 .^{\mathrm{a}}$ ed.

Becker, G. y Murphy, K. (1988), «A Theory of Rational Addiction», The Journal of Political Economy, vol. 96 (4). 
Buchanan, J. y Yoon, Y. (2000) «Symmetric Tragedies Commons and Anticommons», Journal of Law and Economics, vol. 43 (1), 1-14.

Chiang, A. (1984), Fundamental Methods of Mathematical Economics, Singapore, McGrawHill Internacional Edition.

CIS (2005), Tabaquismo y nueva normativa anti-tabaco. Avance de resultados, Estudio número 2627, Madrid, Centro de Investigaciones Sociológicas.

COASE, R. (1960) «The problem of social cost», Journal of Law and Economics, vol. 3 (1), $414-$ 440.

Cropper, M. y OAtes, W. (1992), «Environmental economics: a survey», Journal of Economic Literature, vol. 30 (2), 675-740.

Field, B. y Field, M. (2003), Economía Ambiental, Madrid, McGraw-Hill, 3. ${ }^{\text {e ed. }}$

LEY 28/2005, de 26 de diciembre, de medidas sanitarias frente al tabaquismo y reguladora de la venta, el suministro, el consumo y la publicidad de los productos del tabaco. Madrid, BOE 27 de diciembre de 2005.

LóPEZ-Nicolás, Á. (2004) «Tabaquismo y economía pública», en Jiménez-Ruiz, Fagerström y Díaz-Maroto (eds.), Manual de Tabaquismo, Madrid, Aula Médica Ediciones, capítulo 5.

Pearce, D. y Turner, K. (1995), Economía de los Recursos Naturales y del Medio Ambiente, Madrid, Celeste Ediciones.

PezzeY, J. (1988) «Market mechanisms of pollution control: polluter pays, economic and practical aspects», en K. Turner (ed.), Sustainable Environment Management: Principles and Practices, Londres, Belhaven Press.

Riera, P.; García, D.; Kriström, B. y Brännlund, R. (2005), Manual de Economía Ambiental $y$ de los Recursos Naturales, Madrid, Thomson.

Suranovic, S.; GoldFarb, R. y LeOnard, T. (1999) «An economic theory of cigarrette addiction», Journal of Health Economics, vol. 18 (1), 1-29.

\section{Anexo}

\section{Condiciones analíticas de los escenarios relevantes}

Sean Uma $\mathrm{F}(\mathrm{q})=\mathrm{m}\left(\mathrm{q}^{\mathrm{F}}-\mathrm{q}\right)$ y $\operatorname{Uma} \mathrm{N}^{\mathrm{F}}(\mathrm{q})=\mathrm{n}\left(\mathrm{q}-\mathrm{qN}^{\mathrm{F}}\right)$ la función de utilidad marginal de los individuos fumadores y de desutilidad marginal de los no-fumadores, respectivamente. Aplicando el principio de equimarginalidad se obtiene el óptimo de contaminación $\mathrm{q}^{*}$ :

$$
\mathrm{m}\left(\mathrm{q}^{\mathrm{F}}-\mathrm{q}\right)=\mathrm{n}\left(\mathrm{q}-\mathrm{q}^{\mathrm{NF}}\right) \Rightarrow \mathrm{q}^{*}=\left(\mathrm{m} \mathrm{q}^{\mathrm{F}}+\mathrm{nq}^{\mathrm{NF}}\right) /(\mathrm{m}+\mathrm{n})
$$


Las superficies A, B, C y D en la Gráfica 2 verifican las ecuaciones siguientes:

$$
\begin{aligned}
& \mathrm{A}=\mathrm{n}\left(\mathrm{q}_{0}^{\mathrm{F}}-\mathrm{q}^{\mathrm{NF}}\right)^{2} / 2 \\
& \mathrm{~B}=\left[\mathrm{m}\left(\mathrm{q}^{\mathrm{F}}-\mathrm{q}_{0}^{\mathrm{F}}\right)-\mathrm{n}\left(\mathrm{q}_{0}^{\mathrm{F}}-\mathrm{q}^{\mathrm{NF}}\right)\right]\left(\mathrm{q}^{*}-\mathrm{q}_{0}^{\mathrm{F}}\right) / 2 \\
& \mathrm{C}=\left[\mathrm{n}\left(\mathrm{q}^{\mathrm{NF}}{ }_{0}-\mathrm{q}^{\mathrm{NF}}\right)-\mathrm{m}\left(\mathrm{q}^{\mathrm{F}}-\mathrm{q}^{\mathrm{NF}}\right)\right]\left(\mathrm{q}_{0}^{\mathrm{NF}}{ }_{0}-\mathrm{q}^{*}\right) / 2 \\
& \mathrm{D}=\mathrm{m}\left(\mathrm{q}^{\mathrm{F}}-\mathrm{q}_{0}^{\mathrm{NF}}\right)^{2} / 2
\end{aligned}
$$

A partir de las ecuaciones (A.2), (A.3), (A.4) y (A.5) se obtienen las siguientes relaciones:

$$
\begin{aligned}
& \mathrm{B}>\mathrm{A} \Leftrightarrow \mathrm{m}>\mathrm{m} 1=\mathrm{n}\left[(\mathrm{qF} 0-\mathrm{qNF})\left(\mathrm{q}^{*}-\mathrm{qNF}\right)\right] /\left[(\mathrm{qF}-\mathrm{qF} 0)\left(\mathrm{q}^{*}-\mathrm{qF} 0\right)\right] \\
& \mathrm{C}>\mathrm{D} \Leftrightarrow \mathrm{m}<\mathrm{m} 2=\mathrm{n}\left[(\mathrm{qNF} 0-\mathrm{qNF})\left(\mathrm{qNF} 0-\mathrm{q}^{*}\right)\right] /\left[(\mathrm{qF}-\mathrm{qNF})\left(\mathrm{qF}-\mathrm{q}^{*}\right)\right] \\
& \mathrm{A}+\mathrm{C}>\mathrm{B}+\mathrm{D} \Leftrightarrow \mathrm{m}<\mathrm{m} 3=\mathrm{n}\left[(\mathrm{qF} 0-\mathrm{qNF})\left(\mathrm{q}^{*}-\mathrm{qNF}\right)+\right. \\
& \left.\left(\mathrm{qNF} 0-\mathrm{q}^{*}\right)(\mathrm{qNF} 0-\mathrm{qNF})\right] /\left[(\mathrm{qF}-\mathrm{qNF})\left(\mathrm{qF}-\mathrm{q}^{*}\right)+\right. \\
& \left.\left(\mathrm{q}^{*}-\mathrm{qF} 0\right)(\mathrm{qF}-\mathrm{qF} 0)\right]
\end{aligned}
$$

Las ecuaciones (A.6), (A.7) y (A.8), junto con la Gráfica 2 permiten deducir que $\mathrm{q}^{*}$ es óptimo si y sólo si $\mathrm{m}_{2}>\mathrm{m}>\mathrm{m}_{1}$ (escenario 1$)$, que $\mathrm{q}^{\mathrm{F}}$ es óptimo si y sólo si $\mathrm{m}>$ $\max \left\{\mathrm{m}_{1}, \mathrm{~m}_{2}\right\}$ (escenario 2 ), que $\mathrm{q}^{\mathrm{NF}}$ es óptimo si y sólo si $\mathrm{m}<\min \left\{\mathrm{m}_{1}, \mathrm{~m}_{2}\right\}$ (escenario 3), que $q^{\mathrm{NF}}$ es óptimo si y sólo si $\min \left\{\mathrm{m}_{1}, \mathrm{~m}_{3}\right\}>\mathrm{m}>\mathrm{m}_{2}$ (escenario 4) y que $\mathrm{q}^{\mathrm{F}}$ es óptimo si y sólo si $\mathrm{m}_{1}>\mathrm{m}>\max \left\{\mathrm{m}_{2}, \mathrm{~m}_{3}\right\}$ (escenario 5). 